\title{
Electromagnetic response of layered superconductors with broken lattice inversion symmetry
}

\author{
B. Uchoa ${ }^{1}$, A. H. Castro Neto ${ }^{2}$, and G. G. Cabrera ${ }^{1}$ \\ 1 Instituto de Física "Gleb Wataghin" Universidade Estadual de Campinas (UNICAMP), \\ C. P. 6165, Campinas, SP 13083-970, Brazil \\ 2 Department of Physics, Boston University, \\ 590 Commonwealth Ave., Boston, MA 02215
}

(Dated: July 17, 2003)

\begin{abstract}
We investigate the macroscopic effects of charge density waves (CDW) and superconductivity in layered superconducting systems with broken lattice inversion symmetry (allowing for piezoelectricity) such as two dimensional (2D) transition metal dichalcogenides (TMD). We work with the low temperature time dependent Ginzburg-Landau theory and study the coupling of lattice distortions and low energy CDW collective modes to the superconducting order parameter in the presence of electromagnetic fields. We show that superconductivity and piezoelectricity can coexist in these singular metals. Furthermore, our study indicates the nature of the quantum phase transition between a commensurate CDW phase and the stripe phase that has been observed as a function of applied pressure.
\end{abstract}

PACS numbers: 74.70.Ad, 71.10.Hf, 71.45.Lr

\section{INTRODUCTION}

The quasi-two-dimensional (2D) transition metal dichalcogenides (TMD) $\frac{1}{\underline{1}} 2 \mathrm{H}-\mathrm{NbS}_{2}, 2 \mathrm{H}-\mathrm{NbSe}_{2}, 2 \mathrm{H}-\mathrm{TaS}_{2}$ and $2 \mathrm{H}-\mathrm{TaSe}_{2}$ are layered compounds where superconductivity coexists with a charge density wave (CDW) state $^{2}$. It has been documented experimentally that these materials present anomalous properties such as a decrease of the resistivity and decoupling between planar and c-axis transport in the CDW phase $\mathrm{e}^{3}$, anomalous impurity effects on the superconductivity and non-linear Hall effect ${ }^{4}$, stripe phases ${ }^{5}$, and more recently angle resolved photoemission experiments (ARPES) have shown a quasi-particle lifetime diverging linearly with the energy close to the Fermi surface $e^{\underline{\underline{m}}}$ in contrast to ordinary Fermi liquids where the lifetime diverges quadratically with energy. Some of these properties are similar to the ones observed in high temperature superconductors (HTc $)^{7}$. Contrary to HTc, however, TMD are extremely clean systems and therefore the anomalous behavior is undoubtedly intrinsic and sample independent. Therefore the understanding of the anomalous metallic behavior in these 2D materials may provide clues for the understanding of the anomalous physics that occurs in a broad class of 2D systems where superconductivity occurs.

One of us (AHCN), has proposed recently an unified picture of the CDW and superconducting transitions that can explain some of the anomalies observed experimentally ${ }^{8}$. The theory proposes that the mechanism of superconductivity is related to the pairing of the CDW elementary excitations mediated by acoustic phonons via a piezoelectric coupling. These excitations are Dirac electrons situated in the nodes of a CDW gap, resembling the Fermi surface zone of graphite. In TMD, unlike the case of graphite, the lattice inversion symmetry is broken in the CDW phase. The breaking of the inversion symmetry in TMD, as documented in neu- tron scattering experiments $\frac{9}{}$, allows for a piezoelectric coupling between charge carriers and phonons ${ }^{10}$. Since CDW formation is usually related to nested Fermi surfaces in 1D systems, weak 2D nesting combined with strong variations in the electron phonon coupling due to the tight-binding nature of the electronic orbitals, can be responsible for the origin of the nodal CDW order parameter. This model is able to correctly explain some of the anomalous properties of TMD such as the energy dependence of the quasi-particle lifetime (given by the imaginary part of the Dirac fermion self-energy), the critical dependence of the superconducting transition with the lattice parameters, the reduction of the resistivity, and the metallic behavior in the CDW phase.

However, a criticism has been raised to this theory based on the fact that piezoelectricity normally occurs in insulating systems since metals can screen the internal electric fields 11 . However, Dirac fermions in 2D have a vanishing density of states at the Fermi energy and therefore do not screen electric fields ${ }^{12}$ allowing for the existence of a metallic state (that is, a state with gapless fermionic excitations) and piezoelectricity. Nevertheless, the possibility of coexistence of piezoelectricity and superconductivity is indeed surprising. In such a system it would be possible to generate super-currents by simply squeezing the sample. In this work we show that there is no contradiction between the existence of piezoelectricity and superconductivity in materials where the low-lying excitations are Dirac fermions. We investigate the current and charge fluctuations that would arise in these systems under the view point of the collective modes.

Using path integrals we derive a semi-classical action that describes the coupling between plasmons (responsible for screening) and acoustic phonons to the superconducting order parameter via the piezoelectric coupling. Various well-known regimes are described by this action: (1) in the case of normal electrons without supercon- 
ductivity we recover the well-known results for collective modes and screening in $2 \mathrm{D}$ and $3 \mathrm{D}^{13} ;(2)$ in the case of Dirac fermions without superconductivity we re-obtain the results for collective modes and screening in semimetals like graphite ${ }^{14} ;(3)$ in the absence of piezoelectric coupling, we recover the behavior of an ordinary type II superconductor ${ }^{15}$. However, when we allow the piezoelectric coupling with Dirac fermions and superconductivity, new effects appear in the electromagnetic response. Moreover, our results shed light on the origin of the quantum phase transition between the commensurate CDW phase and the stripe phase in TMD. We also investigate the collective modes that appear when combined with low lying energy bulk plasmons.

The article is organized in the following manner: in section II we discuss the plasmons in layered nodal liquids and specifically in TMD; in section III we develop the semi-classical calculation in general grounds and apply it to layered compounds like TMD in section IV. We have added an appendix where the details of the calculation are presented.

\section{NODAL LIQUID PLASMONS}

The field theory of nodal liquids 16 is built under the basic idea that the nodes of a CDW order parameter lead to two distinct subsystems which correspond to the two components of the Dirac fermion spinor $\Phi_{\sigma}^{\dagger}=\left(\psi_{+\sigma}^{\dagger}, \psi_{-\sigma}^{\dagger}\right)$, with,+- indexing respectively the fermionic particles and anti-particles (holes). The noninteracting low energy Hamiltonian that describes the elementary excitations inside the Dirac cone is:

$$
\mathcal{H}_{D}=\sum_{\sigma} \int_{B Z} \frac{\mathrm{d}^{3} k}{(2 \pi)^{3}} \Phi_{\sigma}^{\dagger}(\mathbf{k}) \hbar\left(v_{F} \sigma_{x} k_{x}+v_{\Delta} \sigma_{y} k_{y}\right) \Phi_{\sigma}(\mathbf{k})
$$

where $\mathbf{k}$ is the momentum, $\sigma_{x, y}$ are Pauli matrices that act in the particle-anti-particle subspace, $v_{F}$ and $v_{\Delta}$ are the anisotropic velocities perpendicular and parallel respectively to the Fermi surface, and $B Z$ is the first Brillouin zone of size $2 \pi / d$ along the $k_{z}$ axis ( $d$ the inter-plane distance). When the chemical potential, $\mu$, intercepts the Dirac point, the Hamiltonian (11) leads to the zero order polarization function 17 at $T=0$, whose complex conjugate is given by:

$$
\Pi^{0 *}(\omega, \mathbf{q})=-\frac{v_{F}}{8 d \hbar v_{\Delta}} \frac{\bar{q}^{2}}{\sqrt{v_{F}^{2} \bar{q}^{2}-\omega^{2}}},
$$

where $\omega$ is the frequency, $\overline{\mathbf{q}}=\vec{q}_{x}+\left(v_{\Delta} / v_{F}\right) \vec{q}_{y}$ the anisotropic in-plane momentum. In the absence of hopping between the planes or interactions with the lattice, the collective excitations of the Dirac fermions are due to the $3 \mathrm{D}$ Coulomb interaction, $V_{0}$, between carriers in different planes: $V_{0}\left(q, k_{z}\right)=2 \pi d e^{2} /\left(\epsilon_{0} q\right) S\left(q, k_{z}\right)$, with a structure factor $S\left(q, k_{z}\right)=\sinh (q d) /\left[\cosh (q d)-\cos \left(k_{z} d\right)\right]$ identical to the layered electron gas (LEG) $\operatorname{case}^{18}(e$ is the electron charge, and $\epsilon_{0}$ the dielectric constant). In the random phase approximation (RPA) the electric susceptibility is given by: $\epsilon\left(\omega, \mathbf{q}, k_{z}\right)=1-V_{0}\left(q, k_{z}\right) \Pi^{0}(\omega, \mathbf{q})$. At the points where the susceptibility vanishes, that is, $\epsilon\left(\omega_{p}\left(\mathbf{q}, k_{z}\right), \mathbf{q}, k_{z}\right)=0$, one obtains the plasmon dispersion, $\omega_{p}\left(\mathbf{q}, k_{z}\right)$. From (2), we notice that no plasmon modes are allowed for Dirac fermions.

The displacement of the chemical potential from the Dirac point generates a pocket around the nodes which drastically changes this picture. The density of states becomes finite at the Fermi surface with Fermi momentum $k_{F}^{*}$, and Fermi energy $E_{F}^{*}=\hbar k_{F}^{*} v_{F}$, giving rise to intra-band excitations in the cone. In this case we recover the optic plasmon $\omega_{p}(q)=\sqrt{\omega_{0}^{2}(\bar{q} / q)^{2}+\left(v_{0} \bar{q}\right)^{2}}$ for the $k_{z}=0$ bulk mode, where $v_{0}$ is the plasmon speed, and the 2D acoustic mode $\omega_{p}(q) \propto \sqrt{\bar{q}^{2} / q}$ for the rest of the plasmon band $0<k_{z} \leq \pi / d$. The intra-band contribution is satisfactorily understood in the context of doped graphite $\frac{14}{\underline{4}}$ where for $v_{F} \bar{q} \leq \omega<v_{F}\left(2 k_{F}^{*}-\bar{q}\right)$ there is gap in the particle-hole continuum of the inter-band excitations (see fig.1) defined by the imaginary part of (2). The optical plasmon is free of Landau damping in the long wavelength limit and its energy is of the order of $E_{F}^{*}$. Notice that in the anisotropic case $\left(v_{F} \neq v_{\Delta}\right)$ the gap in the plasmon spectrum depends on the direction around the pocket Fermi surface.

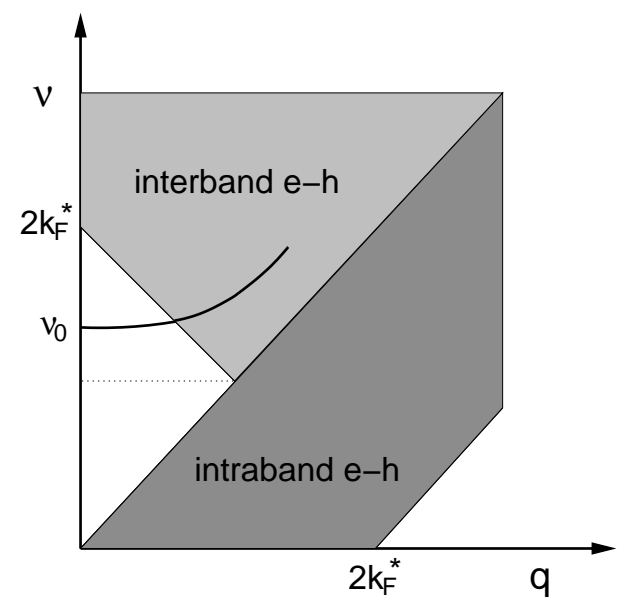

FIG. 1: Schematic drawing of the $k_{z}=0$ bulk plasmon in an isotropic pocket with Fermi momentum $k_{F}^{*} \cdot \quad \nu=\omega / v_{F}$ and $q$ is the in-plane transfer momentum. The shaded areas correspond to the particle-hole continuum due to intra-band and inter-band excitations.

If we also add the piezoelectric electron-phonon coupling 8

$$
\mathcal{H}_{E P}=\gamma \sum_{\sigma} \int \mathrm{d}^{3} x \phi(\mathbf{x}) \Phi_{\sigma}^{\dagger}(\mathbf{x}) \Phi_{\sigma}(\mathbf{x})
$$

due to acoustic phonons (with phonon field $\phi(\mathbf{x})$ ) with energy $\hbar \omega_{\mathbf{q}}$ into the pocket excitations, the RPA electric susceptibility acquires a correction: $\epsilon\left(\omega, \mathbf{q}, k_{z}\right)=$ 


$$
\begin{gathered}
1-\left[V_{0}\left(q, k_{z}\right)+\left(\gamma^{2} / \hbar\right) D^{0}(\omega, \mathbf{q})\right] \Pi^{0}(\omega, \mathbf{q}, \mu) \text { where } \frac{13}{} \\
D^{0}(\omega, \mathbf{q})=\frac{\hbar \omega_{\mathbf{q}}^{2}}{\omega^{2}-\left(\omega_{\mathbf{q}}-i \eta\right)^{2}}
\end{gathered}
$$

is the phonon propagator that affects very little the optical bulk plasmon in the $q \rightarrow 0$ limit.

Besides doping, coherent inter-layer hopping is known to drive a dimensional crossover in direction to a 3D system, leading as well to a pocket formation in a nodal liquid ${ }^{19}$. The energy of the pocket is of the same order of the inter-layer hopping energy, which in TMD $\mathrm{NbSe}_{2}$ and $\mathrm{NbS}_{2}$ (we will drop the $2 \mathrm{H}$ prefix from now on) were calculated in $\sim 0.1 \mathrm{eV}^{20}$. We notice that inter-layer interactions were not taken into account into those band calculations and that this energy could be considerably smaller. More experimental studies are required to investigate the nature of the low energy collective modes in these materials. However, we can consider the plasmon modes in real materials to have a finite energy, like in ordinary metals. Nevertheless, the plasmon frequency will be smaller than in ordinary metals because of the low density of states in the system.

\section{SEMI-CLASSICAL DYNAMICS}

We consider the problem of Dirac fermions described by (11) coupled to lattice vibrations described by the Hamiltonian

$$
H_{P H}=\sum_{n} \frac{\mathbf{P}_{n}^{2}}{2}+\frac{1}{2} \sum_{n, m} K_{n m}\left(\mathbf{R}_{n}-\mathbf{R}_{m}\right)^{2}
$$

where $n$ labels the lattice site $R_{n}^{i}, P_{n}^{i}$ is the lattice momentum operator with $i=x, y, z$ (these operators are canonically conjugated, namely, $\left.\left[P_{n}^{i}, R_{m}^{j}\right]=i \hbar \delta_{i j} \delta_{n m}\right)$, $K_{n m}$ is the coupling matrix. In what follows we will also consider the coupling of the Dirac fermions and lattice degrees of freedom to a classical electromagnetic field described by a vector potential field, $\mathbf{A}(\mathbf{r})$, and electromagnetic field tensor, $F^{\mu \nu}=\partial^{\mu} A^{\nu}-\partial^{\nu} A^{\mu}\left(\partial^{\mu}=\partial / \partial x_{\mu}\right.$ with $\mu, \nu=0, i)$ with electromagnetic energy density:

$$
E_{e l}=-\frac{1}{16 \pi} F_{\mu \nu} F^{\mu \nu}
$$

where $F_{0 j}=-E_{j}$ is the electric field strength (we use the metric $\left.g_{\mu, \nu}=(1,-1,-1,-1)\right)^{21}$. In a piezoelectric the electric field couples to the lattice distortion, $X_{n}^{i}=$ $R_{n}^{i}-R_{n+1}^{i}$, via the piezoelectric tensor, $\Delta_{j}^{i}$, by:

$$
H_{P}=\sum_{n} \Delta^{i}{ }_{j} E_{i} X_{n}^{j}
$$

where repeated indices are to be summed. The coupling between Dirac fermions and lattice vibrations is given by (3). The full problem can be written in path integral form for the generating functional of the problem:

$$
Z=\int D \bar{\psi} D \psi \int D \mathbf{X} \exp \left\{\frac{i}{\hbar} \int d t \int d \mathbf{r} \mathcal{L}\left[\psi, \mathbf{X}, A_{\mu}\right]\right\}
$$

where $\mathcal{L}$ is the Lagrangian (real time) of the problem $(\bar{\psi}$, $\psi$ are Grassmann variables).

Let us consider first the case of the normal (nonsuperconducting) state. We are only interested in the long wavelength physics of the electronic problem which, as argued in the previous section, is described by the plasmon mode. The plasmon mode can be separated from the particle-hole continuum using the formalism developed by Bohm and Pines 22 and the Lagrangian of the problem reduces to:

$$
\begin{aligned}
\mathcal{L}_{N}= & \mathcal{L}_{\text {el }}+\mathcal{L}_{\text {plasmon }}+\mathcal{L}_{\text {phonon }}+\mathcal{L}_{\text {piez }} \\
= & -\frac{1}{16 \pi} F_{\mu \nu} F^{\mu \nu}-\frac{1}{c} j_{N}^{\mu} A_{\mu}+\frac{1}{2} \Omega\left[\omega_{p}^{-2}\left(\partial_{t} \rho_{N}\right)^{2}-\rho_{N}^{2}\right] \\
& +\frac{1}{2} \kappa\left[\left(\partial_{t} X^{i}\right)^{2}-v_{p h}^{2}\left(\partial_{i} X^{i}\right)^{2}\right]+\Delta^{i}{ }_{j} X_{i} F^{0 j},
\end{aligned}
$$

where $\Omega \approx 4 \pi / k_{c}^{2}$, with $k_{c}$ the $3 \mathrm{D}$ electron screening wave vector (for an isotropic metal $k_{c}=\sqrt{3 \pi n e^{2} /\left(2 E_{F}\right)}$ where $n$ is the $3 \mathrm{D}$ electron density and $E_{F}$ the Fermi energy ${ }^{22}$ ), $\kappa$ is the lattice mass density, $v_{p h}$ is the sound velocity, $j_{N}^{0} / c=\rho_{N}$ is the density of normal electrons and $j_{N}^{i}$ is the normal Ohmic current density (in our notation, $\partial^{0}=c^{-1} \partial_{t}$ is the time derivative normalized by the speed of light $c$ ).

The collective properties of the electrons in the normal phase are the same of the electrons in the superconducting phase, since the opening of the superconducting gap does not affect the real part of the electronic polarization function, $\Pi(\mathbf{q})$, in the long wavelength limit 23 . In fact, within RPA one can easily show in the context of BCS theory that $\operatorname{Re} \Pi(\mathbf{q})$ remains essentially unchanged until the superconducting gap is of the order of the Fermi energy, when the BCS pairing approximation becomes invalid ${ }^{24}$. In other words, for small $q$ the plasmon is not sensitive to the superconductor phase transition. Thus, normal and superconducting electrons screen electric fields exactly in the same way and therefore enter at equal footing in the Lagrangian. Thus, irrespective of the phase, normal or superconducting, $\rho_{N}$ in (8) can be replaced by the total electron density $\rho=\rho_{N}+\rho_{s}$, where $\rho_{s}$ is the density of superconducting electrons $\left(\rho_{s}=0\right.$ in the normal state). Normal currents, however, are due to the quasi-particle excitations while the supercurrents, $j_{s}^{\mu}$, are due to the ground state of the condensate ${ }^{25}$. Thus, in the superconducting state the vector potential only couples to the normal currents. At finite temperature, $T$, the number of normal and superconducting electrons is not conserved. For simplicity, we have chosen to work at $T=0$, where all the electrons are in the condensate and the total current is $j^{\mu}=j_{s}^{\mu}$.

In the superconducting phase we can introduce the superconducting order parameter, $\Psi$, via a standard Hubbard-Stratanovich transformation and trace over the electrons ${ }^{26}$. The generating functional reads:

$$
\begin{aligned}
Z & =\int D \bar{\Psi} D \Psi \int D \mathbf{X} \exp \left\{\frac { i } { \hbar } \int d t \int d \mathbf { r } \left(\mathcal{L}_{N}[\rho, \mathbf{X}, \mathbf{A}]\right.\right. \\
& \left.\left.+\mathcal{L}_{G L}[\Psi, \mathbf{A}]\right)\right\}
\end{aligned}
$$


where $\mathcal{L}_{G L}$ is the Ginzburg-Landau Lagrangian. In order to describe the macroscopic current-charge fluctuations we must also include a time dependence in the superconductor order parameter. This is not an obvious task but it can be done in two special limits: close to $T=0$, and near the critical temperature ${ }^{27}$. In the later, the validity of the time-dependent Gorkov equations expansion in the gap, $\Delta_{s}$, require frequencies higher than the binding energy of the Cooper pairs, $\hbar \omega \gg \Delta_{s}$. In this limit the fluctuations have enough energy to break the Cooper pairs and convert them into single particle excitations, leading to a diffusive regime. As we mentioned above, we will work in the opposite limit $T=0$ where the hydrodynamic description at $\hbar \omega<\Delta_{s}$ is rigorously valid. We introduce the low-temperature time-dependent Ginzburg-Landau Lagrangian ${ }^{27,28}$,

$$
\begin{aligned}
\mathcal{L}_{G L}= & -\alpha|\Psi|^{2}-\beta|\Psi|^{4}-\frac{1}{2 m^{\star}}\left|\left(\frac{\hbar}{i} \nabla-\frac{e^{\star}}{c} \mathbf{A}\right) \Psi\right|^{2} \\
& +\frac{1}{2 m^{\star} v_{s}^{2}}\left|\left(\frac{\hbar c}{i} \partial^{0}+e^{\star} \phi\right) \Psi\right|^{2} \cdot
\end{aligned}
$$

which is specially suitable for type II superconductors, where the penetration length is much larger than the coherence length (in (10) $\phi$ is the electrostatic potential and all the other symbols are standard $\left.{ }^{15}\right)$. In the clean limit, $v_{s}$ is typically of the order of the Fermi velocity $v_{F}$. Under the assumption that the fields vary much slower than the coherence length, we assume that the magnitude of the superconductor order parameter is constant and therefore all the fluctuations come from the superconducting phase, $\varphi(t, \mathrm{x})$. Thus, the order parameter is written as:

$$
\Psi(\mathrm{x})=\Psi_{0} \mathrm{e}^{i \varphi(t, \mathrm{x})} .
$$

Despite the gauge invariance of (10), we conveniently assume the transverse gauge, $\nabla \cdot \mathbf{A}=0$, in what follows. Notice, however, that $\mathcal{L}_{N}+\mathcal{L}_{G L}$ is not complete because the fields and the supercurrents remain decoupled. This problem is solved by assuming the validity of the nonhomogeneous Maxwell equations. The Poisson equation is clearly a constraint between the density of superconducting electrons and the electrostatic potential,

$$
g(t, \mathbf{x}) \equiv \nabla^{2} \phi+4 \pi \rho_{s}=0
$$

and has to be enforced using a Lagrange multiplier $\Lambda\left(x^{\mu}\right)$ in final Lagrangian.

In the semi-classical regime, $\hbar \rightarrow 0$, the behavior of the fields comes from the minimal action principle

$$
\delta S=\delta \int \mathrm{d}^{4} x\left(\mathcal{L}_{N}+\mathcal{L}_{G L}+\Lambda g\right)=\delta \int \mathrm{d}^{4} x \mathcal{L}=0
$$

with respect to the variables $A^{\mu}, \rho_{s}, X^{i}, \varphi$ and $\Lambda$. By minimizing with respect to $A^{\mu}$ and $\Lambda$, we obtain the two non-homogeneous Maxwell's equations,

$$
\nabla^{2} \phi+4 \pi \rho_{s}=0
$$

$$
-\square \mathbf{A}-\partial_{0} \nabla \phi+\frac{4 \pi}{c} \mathbf{j}_{s}=0
$$

with the supercurrent $j_{s}^{\mu}$ given by

$$
\begin{gathered}
\rho_{s}=-\frac{e^{\star} \Psi_{0}^{2}}{m^{\star} v_{s}^{2}}\left(\hbar c \partial_{0} \varphi+e^{\star} \phi\right)-\delta \nabla \cdot \mathbf{X}-\nabla^{2} \Lambda \\
\mathbf{j}_{s}=\frac{e^{\star} \Psi_{0}^{2}}{m^{\star}}\left[\hbar \nabla \varphi-\frac{e^{\star}}{c} \mathbf{A}\right]+c \delta \partial_{0} \mathbf{X} .
\end{gathered}
$$

For simplicity we will assume that $\Delta^{i}{ }_{j}=\delta \delta_{i j}$ is a diagonal and isotropic tensor. The minimization with respect to the other fields completes the set of equations:

$$
\begin{gathered}
\frac{1}{4 \pi} \Omega\left(\omega_{p}^{-2} \partial_{t}^{2} \rho_{s}+\rho_{s}\right)=\Lambda \\
\nabla^{2} \varphi-\frac{c^{2}}{v_{s}^{2}}\left(\partial^{0}\right)^{2} \varphi-\frac{e^{\star} c}{\hbar v_{s}^{2}} \partial^{0} \phi=0 \\
\kappa\left(\partial_{t}^{2} \mathbf{X}-v_{p h}^{2} \nabla^{2} \mathbf{X}\right)-\delta\left(\nabla \phi+\partial^{0} \mathbf{A}\right)=0 .
\end{gathered}
$$

Substituting the supercurrent in the continuity equation, $\partial_{\mu} j_{s}^{\mu}=0$, we find that

$$
c \partial_{0} \rho_{s}+\partial_{i} j_{s}^{i}=-c \partial_{0} \nabla^{2} \Lambda=0,
$$

and therefore, in the absence of external currents $\Lambda$ is either a function of space or of time, but not of both. If $\Lambda$ depends on time, then $\rho$ is a stationary time dependent homogeneous (or non-periodic) distribution because of (16), what obviously violates the charge conservation. Therefore, we conclude that $\partial_{0} \Lambda=0$.

The Lagrange multiplier defines two distinct classes of solutions for the charge density: $(i)$ screening-like when $\Lambda(\mathbf{x}) \neq 0$, and (ii) plasmon-like when $\Lambda \equiv 0$. In the first case, $\rho(\omega, \mathbf{k}) \propto f(\mathbf{k}) \delta(\omega)$, where $f(\mathbf{k})$ depends on the boundary conditions, has no dynamics and describes the physical response to a boundary perturbation such as a static squeeze of the crystal or the introduction of a charge probe. In case $(i i)$, the electrons can oscillate freely with the plasmon frequency, allowing the existence of normal modes.

\section{COLLECTIVE MODES}

We consider the problem of a layered solid (such as the TMD) made by an infinite number of weakly interacting planes. In the continuum limit this problem becomes an effective 3D model with spatial anisotropy in the direction perpendicular to the planes, say the $z$ axis. It is convenient to define the diagonal anisotropy tensor $\tau_{j}^{i}=(1,1, \tau)$, where $\tau$ is the anisotropy parameter, 
(we introduce the notation $\tilde{\partial}^{i} \equiv \tau^{i}{ }_{j} \partial^{j}$ and $\tilde{A}^{i} \equiv \tau^{i}{ }_{j} A^{j}$ ) and rewrite the Ginzburg-Landau Lagrangian as a timedependent Lawrence-Doniach mode ${ }^{15}$ :

$$
\begin{aligned}
\mathcal{L}_{G L}= & -\alpha|\Psi|^{2}-\beta|\Psi|^{4}-\frac{1}{2 m^{\star}}\left|\left(\frac{\hbar}{i} \tilde{\partial}^{i}-\frac{e^{\star}}{c} \tilde{\mathbf{A}}\right) \Psi\right|^{2} \\
& +\frac{1}{2 m^{\star} v_{s}^{2}}\left|\left(\frac{\hbar c}{i} \partial^{0}+e^{\star} \phi\right) \Psi\right|^{2} .
\end{aligned}
$$

The model leads to the anisotropic version of equation (17),

$$
\tilde{\nabla} \cdot \tilde{\nabla} \varphi-\frac{c^{2}}{v_{s}^{2}}\left(\partial^{0}\right)^{2} \varphi-\frac{e^{\star} c}{\hbar v_{s}^{2}} \partial^{0} \phi-\frac{e^{\star}}{\hbar c} \tilde{\nabla} \cdot \tilde{\mathbf{A}}=0,
$$

and to the appearance of Josephson currents between the planes, represented by an anisotropic supercurrent:

$\mathbf{j}_{s}(t, \mathbf{x})=\frac{e^{\star} \Psi_{0}^{2}}{m^{\star}}\left[\hbar \tilde{\nabla} \varphi(t, \mathbf{x})-\frac{e^{\star}}{c} \tilde{\mathbf{A}}(t, \mathbf{x})\right]+c \delta \partial_{0} \vec{X}(t, \mathbf{x})$.

From now on, we use an overhead symbol to represent the in-plane vectors $\vec{x}, \vec{q}, \vec{X}, \vec{\nabla}$ and etc. In our notation, we define $\vec{q}$ as the in-plane component of the momentum $\mathbf{k}$, such that $\mathbf{k}=\left(q, k_{z}\right)$ and $\tilde{\mathbf{k}}=\left(q, \tau k_{z}\right)$.

In highly anisotropic layered compounds the absence of piezoelectricity along the $z$ axis is justified by the weak distortions of the ions in this direction. The $z$-component of the sound velocity is also very small due to the weak elastic coupling between planes. In the $v_{p h z} / v_{p h} \rightarrow 0$ limit, the phonon dispersion is $\omega_{p h}=v_{p h} q$ and the inplane phonon equation (18) has a cylindrical symmetry

$$
\begin{aligned}
\kappa\left(-\omega^{2}+\omega_{p h}^{2}\right) \vec{X}(\omega, \mathbf{k}) \\
-i \delta\left[\vec{q} \phi(\omega, \mathbf{k})+\frac{\omega}{c} \vec{A}(\omega, \mathbf{k})\right]=0 .
\end{aligned}
$$

Given the plasmon frequency, $\omega_{p}(\mathbf{k})$, the equation for the charge density in Fourier space becomes:

$$
\Omega\left[-\omega^{2}+\omega_{p}^{2}(\mathbf{k})\right] \rho_{s}(\omega, \mathbf{k})=8 \pi^{2} \omega_{p}^{2}(\mathbf{k}) \Lambda(\mathbf{k}) \delta(\omega) .
$$

The screening-like solution is:

$$
\rho_{s}(\omega, \mathbf{k})=8 \pi^{2} \frac{\Lambda(\mathbf{k})}{\Omega} \delta(\omega),
$$

for a non-zero $\Lambda(\mathbf{k})$. After a straightforward calculation, the simultaneous solution of equations (12)-(14), (20) - (22) and (24) yields $\rho_{s}(\omega, \mathbf{k})=\Lambda_{0}(\hat{\mathbf{k}}) \delta\left(k^{2}+k_{0}^{2}\right)$, for some $\Lambda_{0}$ function, with the characteristic momentum

$$
k_{0}^{2}=4 \pi\left(\frac{e^{\star 2} \Psi_{0}^{2}}{m^{\star} v_{s}^{2}}-\frac{\delta^{2}}{\kappa v_{p h}^{2}}\right) .
$$

The $k_{0}^{2}>0$ case leads to exponentially decaying solutions that describe the screening induced by proper boundary conditions like the squeeze or shear of the crystal. If the piezoelectric coupling is larger than a critical value $\left(k_{0}^{2}<0\right)$, the screening is suppressed and a quasi-static charge modulation should be observed, in such way to minimize the elastic energy. This analysis is confirmed by introducing an external charge probe $Q$ at the origin: we re-obtain the well known Thomas-Fermi result 13 ,

$$
\rho_{s}(\omega, \mathbf{k})=-2 \pi \frac{Q k_{0}^{2}}{k^{2}+k_{0}^{2}} \delta(\omega),
$$

which gives a screened potential for $k_{0}^{2}>0$ and recovers the metallic case when the cut-off of the in-plane band dispersion $s$ (of the order of inverse of the in-plane lattice spacing) is taken to infinity.

For a finite cut-off $s$ we have various cases. When $k_{0}>s$, the screening is limited to the direction perpendicular to the planes. When $k_{0}^{2}<0$ and $\left|k_{0}\right|<s$, the system does not show any screening since the potential decays like $\cos \left(\left|k_{0}\right| r\right) / r$ for large $r$. The last possible case, $k_{0}^{2}<0$ and $\left|k_{0}\right|>s$ is not physical in this theory and gives a purely imaginary response. We verify that the phase of the superconducting order parameter is free of fluctuations and satisfies the zero-field vortex equation $(\vec{\nabla})^{2} \varphi=0$ in the whole $\Lambda \neq 0$ class.

The collective modes follow from a slightly different calculation. We start from the plasmon solution of (23),

$$
\rho_{s}(\omega, \mathbf{k})=\rho_{0}(\mathbf{k}) \delta\left(\omega-\omega_{p}(\mathbf{k})\right),
$$

where $\rho_{0}(\mathbf{k})$ is a function which depends not only on the boundary conditions but also on the initial conditions driven by the perturbation. In the non-relativistic limit $v_{F} / c \rightarrow 0$, the field solutions in the momentum space are (the details of the calculation are given in the Appendix):

$$
\rho_{s}(\omega, \mathbf{k})=\rho(\hat{q}) \delta\left(q-q_{0}\right) \delta\left(k_{z}\right) \delta\left(\omega-\omega_{p}(\mathbf{k})\right),
$$

$$
\phi(\omega, \mathbf{k})=4 \pi \frac{\rho_{s}(\omega, \mathbf{k})}{k^{2}}
$$

$$
\begin{gathered}
\varphi(\omega, \mathbf{k})=4 \pi i \frac{\omega e^{\star}}{\hbar} \frac{\rho_{s}(\omega, \mathbf{k})}{k^{2}} \frac{1}{\omega^{2}-v_{s}^{2} \tilde{k}^{2}}, \\
\vec{X}(\omega, \mathbf{k})=4 \pi \frac{\delta}{i \kappa} \frac{\vec{q}}{k^{2}} \frac{\rho_{s}(\omega, \mathbf{k})}{\left(\omega^{2}-\omega_{p h}^{2}\right)},
\end{gathered}
$$

$$
\mathbf{A}(\omega, \mathbf{k})=4 \pi \frac{\omega}{c} \vec{q} \frac{\rho_{s}(\omega, \mathbf{k})}{k^{4}} \mathcal{D}(\omega, \mathbf{k})
$$

where we have labeled

$$
\mathcal{D}(\omega, \mathbf{k})=1-4 \pi \frac{e^{\star 2} \Psi_{0}^{2}}{m^{\star}} \frac{1}{\omega^{2}-v_{s}^{2} \tilde{k}^{2}}+4 \pi \frac{\delta^{2}}{\kappa} \frac{1}{\omega^{2}-v_{p h}^{2} q^{2}} .
$$


Equation (28) represents the bulk plasmon mode $k_{z}=0$, which prevails over the rest of the plasmon band. This is in agreement with a general result valid for a stack of layers coupled by Coulomb interactions and by coherent hopping terms between adjacent planes. The presence of inter-layer charge transfer induces a dimensional crossover to a $3 \mathrm{D}$ system, which dominates the long wavelength spectrum. As we show in the end of the Appendix, this effect is conditioned to the assumption that $\tau \neq 0$. The inverse of the in-plane modulation scale $q_{0}$ is given by the equation $\mathcal{D}\left(\omega_{p}, q_{0}\right)=0$.

Noting that at $T=0$ the electronic density $n$ is twice the density of Cooper pairs $\Psi_{0}^{2}$, we realize that the quantity $4 \pi e^{\star 2} \Psi_{0}^{2} / m^{\star}$ can be conveniently written in the form of the expression that gives the square of the plasma frequency, $\Omega_{p}^{2}=4 \pi e^{2} n / m$. In general grounds, $n$ is given by the sum rule $\frac{15}{}$

$$
\omega_{p}^{2}(k=0)=\frac{2}{\pi} \int_{0}^{\infty} \mathrm{d} \omega \omega \operatorname{Im} \epsilon(\omega, k)=4 \pi \frac{e^{2} n}{m} f(\bar{q} / q) .
$$

where $\operatorname{Im} \epsilon$ is the imaginary part of the electronic susceptibility, which is given in RPA by $\operatorname{Im} \epsilon(\omega, \mathbf{k})=$ $-V_{0}\left(q, k_{z}\right) \operatorname{Im} \Pi^{0}(\omega, \vec{q}, \mu)$ and $f(\bar{q} / q)$ is the anisotropy function due to the shape of the Fermi surface, with $f(\bar{q} / q) \equiv 1$ in the isotropic case. For an anisotropic nodal liquid with a small pocket we find that $f(\bar{q} / q)=\bar{q}^{2} / q^{2}$, as it may be easily checked by replacing the leading intraband polarization function ${ }^{14}$

$$
\operatorname{Im} \Pi^{0}(\omega, \vec{q}, \mu) \stackrel{q / k_{F}^{*} \ll 1}{\longrightarrow}-\frac{k_{F}^{*}}{\pi \hbar v_{\Delta} d} \frac{\omega}{\sqrt{v_{F}^{2} \bar{q}^{2}-\omega^{2}}}
$$

into (33), giving

$$
4 \pi \frac{e^{\star 2} \Psi_{0}^{2}}{m^{\star}}=\omega_{0}^{2},
$$

with $\omega_{0}=\sqrt{2 k_{F}^{*} v_{F}^{2} e^{2} /\left(d \epsilon_{0}^{\prime} \hbar v_{\Delta}\right)}$, where $\epsilon_{0}^{\prime}$ is the background susceptibility renormalized by the small interband terms ${ }^{14}$. To simplify the analysis we consider the isotropic case only, where the rigid $\Psi_{0}$ approximation is more rigorous. Using the $k_{z}=0$ bulk plasmon mode $\omega_{p}^{2}=\omega_{0}^{2}+\left(v_{0} q\right)^{2}$, we find that

$$
q_{0}=\sqrt{b-\sigma(b) \sqrt{b^{2}-\frac{g \omega_{0}^{2}}{\left(v_{s}^{2}-v_{0}^{2}\right)\left(v_{p h}^{2}-v_{0}^{2}\right)}}},
$$

where $\sigma$ is a sign function, $b=\left(g+\omega_{0}^{2}\right) /\left[2\left(v_{p h}^{2}-v_{0}^{2}\right)\right]$ and

$$
g=4 \pi \frac{\delta^{2}}{\kappa}
$$

is the effective piezoelectric coupling.

Noting that $v_{s}>v_{p h}$, because the ions are much slower than the electrons, we identify two distinct cases, (1) $v_{0}>$ $v_{s}$ and $(2) v_{0}<v_{s}$. The normal modes are clearly absent in the regime (1) for any value of $g$. On the other hand, these modes are possible in the regime (2) for non zero $g$. Naturally, they would not be physical for $g<\left(v_{p h}^{2} / v_{s}^{2}\right) \omega_{0}^{2}$, where the interactions are screened.

The clean limit of the superconductor gives exactly $v_{s}=v_{F} / \sqrt{3}$. In the particular case of an isotropic nodal liquid with a small pocket, the intra-band bulk plasmon disperses with $v_{0}=(\sqrt{3} / 2) v_{F} \frac{14}{14}$ and therefore it corresponds to the regime (1) mentioned above. This is also the case of the metal $\left(v_{0}=\sqrt{3 / 5} v_{F}\right)$, and of doped graphite $\left(v_{0}=(\sqrt{3} / 2) v_{F}\right)$. Therefore, in the particular case of TMD, we should not observe any essential differences in the plasmon modes in comparison to the LEG (where $g=0$ ) because of the piezoelectricity.

\section{A. Experimental results}

There is a large amount of experimental literature dealing with the observation of commensurate (CDW) or incommensurate charge order in TMD. Neutron and x-ray diffraction measurements in $\mathrm{TaSe}_{2}$ and $\mathrm{NbSe}_{2}$ reveal the existence of Bragg peaks at incommensurate wave vectors $\mathbf{Q}_{i}=\left(1-\delta_{i}\right) \mathbf{b}_{i} / 3$, where $\mathbf{b}_{i}\left(\left|\mathbf{b}_{i}\right|=4 \pi /(\sqrt{3} a)\right)$ are the three reciprocal vectors with hexagonal symmetry, $a$ is the lattice spacing and $\delta_{i} \lesssim 0.02$ is the incommensurability ${ }^{5.9}$. This state is called a triple CDW phase.

In $\mathrm{TaSe}_{2}$, the phase diagram temperature vs. pressure, $P$, is very ich $^{29}$ with three different phases: (1) a high temperature hexagonal incommensurate CDW phase $(\mathrm{HCDW})$ where the three ordering vectors have $\delta_{i} \neq 0(i=1,2,3) ;(2)$ an incommensurate stripe phase where one ordering vector is incommensurate, say $\delta_{1} \neq 0$, but the other two are commensurate, $\delta_{2,3}=0 ;(3)$ a commensurate CDW phase (CCDW) where $\delta_{i}=0(i=$ $1,2,3)$. The transition from the undistorted phase (normal) to HCDW occurs at a pressure independent temperature, $T_{N-H} \approx 120 \mathrm{~K}$, up to pressures of $4.5 \mathrm{GPa}$; the transition between HCDW to stripe phase $T_{H-S} \approx 110 \mathrm{~K}$ is also roughly pressure independent; the transition between stripe phase and CCDW, $T_{S-C}(P)$, is highly pressure dependent and vanishes at $P=P_{c} \approx 1.8 \mathrm{GPa}$. Thus, there is a quantum phase transition $(T=0)$, as a function of pressure at $P=P_{c}$. By further application of pressure there is a reentrant CCDW phase that has not been fully studied and will be not discussed here. We will concentrate on the nature of the quantum critical point (QCP) at $P=P_{c}$.

The nature of the stripe phase has been discussed by McMillan ${ }^{30}$ and others ${ }^{31}$ from the phenomenological point of view as a result of the formation of topological defects of the complex CDW order parameter. This stripe phase can be easily observed with electron microscopy ${ }^{32}$. The microscopic nature of this phase is still unknown but our results here suggest that it may have its origins on the piezoelectric coupling in the solid. Indeed, we have found that there is a charge modulation in these materials when $k_{0}^{2}<0$ (see eq.(26)). 
This modulation can be thought as an incommensuration $\delta_{i}=3 \sqrt{-k_{0}^{2}} /\left|\mathbf{b}_{i}\right|$. Equivalently, using definition (35) there is a critical coupling constant $g_{c}$ so that an extra charge modulation appears $\left(\delta_{i}>0\right)$ when $k_{0}^{2}<0$ or

$$
g>g_{c}=4 \pi \frac{v_{p h}^{2}}{v_{s}^{2}} \frac{e^{\star 2} \Psi_{0}^{2}}{m^{\star}} .
$$

Recall that $\operatorname{TaSe}_{2}\left(T_{c} \approx 0.1 K\right)$ and $\mathrm{NbSe}_{2}\left(T_{c} \approx 8.3 K\right)$ are both superconductors 1 whose $T_{c}$ increase under application of pressure ${ }^{33}$ and therefore $\Psi_{0} \neq 0$ at $T=0$. Notice that both the piezoelectric coupling, $\delta$, as well as the sound velocity, $v_{p h}$, are also monotonically increasing functions of pressure. If we assume that in $\mathrm{TaSe}_{2}$ we have $g<g_{c}$ at $T=0$ and ambient pressure, then the quantum phase transition can occur as a function of pressure as long as $g / v_{p h}^{2}$ is an increasing function of pressure. In $\mathrm{NbSe}_{2}$, however, the system is always incommensurate indicating that $g>g_{c}$ even at ambient pressure. Since the $T=0$ phases seem to be directly connected with the stripe phases that are observed at finite temperature we can immediately conclude that the existence of these stripe phases have to do with the piezoelectric coupling in these materials. Besides, recent experiments report a significant electrostatic modulation of $T_{c}$ in epitaxial bilayers composed by a $\mathrm{HT}_{c}$ cuprate and a polarized insulator deposited on it ${ }^{34}$. The connections between TMD and $\mathrm{HT}_{c}$ are remarkable and we believe that this experimental result could be numerically calculated by relaxing the rigidity in the amplitude $\Psi_{0}$ of the superconductor order parameter. In addition, we suggest that similar electrostatic devices combined with neutron scattering could test experimentally the role of the piezoelectricity in TMD stripe phases.

Neutron scattering measurements have shown the softening of the phonon optical mode $\Sigma_{1}$ in $\mathrm{NbSe}_{2}$ and $\mathrm{TaSe}_{2}$ at the $\mathbf{Q}_{i}$ position for a wide range of temperatures 9 . This behavior was interpreted as a coupling of the optical phonons with the charge order. Constant- $\mathbf{Q}$ scans at $\mathbf{Q}_{i}$ have shown that the phonon energy gap in both cases is of the order of $10 \mathrm{meV}^{9}$. The coupling of the plasmons with the lattice vibrations in the present theory drives the long wavelength phonons to lock their frequency with the frequency of the $k_{z}=0$ bulk plasmon. In comparison to the metallic case where the plasmon modes are rigid (in the sense that their wavelength has exactly the size of the system), we have found that the increase of the elastic energy due to the piezoelectric coupling may give rise to elastic plasmons which oscillate in resonance with the optical phonon mode. Despite not observable in TMD, we believe that this effect would be the macroscopic manifestation of the plasmon-phonon resonance observed experimentally in these materials.

\section{CONCLUSIONS}

We have investigated the collective excitations of the electrons in a layered superconductor by a semi-classical calculation in the continuum limit of a highly anisotropic material. This procedure is analogous to the LawrenceDoniach effective model for an infinite stack of layers. Despite the evident interest in TMD, the calculation is sufficiently general and be could be applied to any superconducting layered compound at zero temperature with broken lattice inversion symmetry.

We have demonstrated from the electrodynamic point of view that superconductivity and piezoelectricity can coexist. Metallic screening is observed when the effective piezoelectric coupling $g$ is smaller than a critical value $g_{c}$, with the Thomas-Fermi momentum reduced by the increase of $g$. Above the critical coupling, the system is not screened and a long-range charge modulation is expected to appear as the response to a local quasi-static charge unbalance, which can be created by squeezing the crystal. We have shown that piezoelectricity is possibly related to the mechanism behind the stripe formation in TMD. Besides, we have investigated the existence of zero temperature normal modes arising in the presence of low energy bulk plasmons, which dominate the spectrum of collective excitations. We have also conjectured that the $k_{z}=0$ plasmon mode behind these excitations comes from the contribution of the intra-band excitations of a pocket opened around the nodes of TMD by low energy coherent hopping terms between adjacent planes. These pockets could be also generated by doping TMD with intercalating materials.

\section{Acknowledgments}

A. H. C. N. thanks C. Varma for stimulating this work. We thank A. J. Leggett for many discussions on the problem of screening in metals and superconductors. B.U. and G.G.C. thank E. Miranda and Y. Copelevich for many helpfull discussions. They also acknowledge Fundação de Amparo à Pesquisa do Estado de São Paulo (FAPESP), Brazil, project number 00/06881-9, for financial support. B.U. thanks the Dept. of Physics at Boston University for the hospitality.

\section{APPENDIX}

Here we derive in detail the calculation of equations (28) - (32). Starting from the plasmon-like solution

$$
\rho_{s}(\omega, \mathbf{k})=\rho_{0}(\mathbf{k}) \delta\left(\omega-\omega_{p}(\mathbf{k})\right)
$$

for a general function $\rho_{0}(\mathbf{k})$ and replacing it in the the Poisson equation (12), we get

$$
\phi(\omega, \mathbf{k})=4 \pi \frac{\rho_{0}(\mathbf{k})}{k^{2}} \delta\left(\omega-\omega_{p}(\mathbf{k})\right)
$$


Next we separate the $z$-component of (13) and (21),

$$
\begin{gathered}
\frac{\omega}{c} k_{z} \phi(\omega, \mathbf{k})+\left(\frac{\omega^{2}}{c^{2}}-k^{2}-\frac{4 \pi}{c^{2}} \tau \frac{e^{\star 2} \Psi_{0}^{2}}{m^{\star}}\right) A_{z}(\omega, \mathbf{k}) \\
+\frac{4 \pi}{c} \frac{e^{\star} \Psi_{0}^{2}}{m^{\star}} i \tau k_{z} \hbar \varphi(\omega, \mathbf{k})=0
\end{gathered}
$$

and the $\varphi$ equation (20)

$$
\begin{aligned}
\left(-\tilde{k}^{2}+\frac{\omega^{2}}{v_{s}^{2}}\right) \varphi(\omega, \mathbf{k}) & \\
& -i \frac{e^{\star}}{\hbar c}\left(\frac{c \omega}{v_{s}^{2}} \phi(\omega, \mathbf{k})+\tilde{\mathbf{k}} \cdot \tilde{\mathbf{A}}(\omega, \mathbf{k})\right)=0 .
\end{aligned}
$$

Noting that $\tilde{\mathbf{k}} \cdot \tilde{\mathbf{A}}=k_{z}\left(\tau^{2}-1\right) A_{z}$, for non-identically zero $\rho_{0}(\mathbf{k})$ we find

$$
\begin{aligned}
& A_{z}(\omega, \mathbf{k})=-\frac{4 \pi}{c} \frac{\omega k_{z}}{k^{2}} \frac{\rho_{0}(\mathbf{k})}{\mathcal{F}(\omega, \mathbf{k})} \delta\left(\omega-\omega_{p}(\mathbf{k})\right) \\
& \quad \times\left[1-4 \pi \frac{e^{\star 2} \Psi_{0}^{2}}{m^{\star} v_{s}^{2}} \frac{\tau}{\mathcal{G}(\omega, \mathbf{k})}\left(1-\frac{v_{s}^{2}}{c^{2}} \frac{\left(\tau^{2}-1\right) k_{z}^{2}}{\mathcal{F}(\omega, \mathbf{k})}\right)\right](
\end{aligned}
$$

and

$$
\begin{aligned}
\varphi(\omega, \mathbf{k})= & 4 \pi i \frac{\omega e^{\star}}{\hbar v_{s}^{2}} \frac{\rho_{0}(\mathbf{k})}{k^{2}} \frac{1}{\mathcal{G}(\omega, \mathbf{k})} \delta\left(\omega-\omega_{p}(\mathbf{k})\right) \\
& \times\left[1-\left(\tau^{2}-1\right) \frac{v_{s}^{2}}{c^{2}} \frac{k_{z}^{2}}{\mathcal{F}(\omega, \mathbf{k})}\right],
\end{aligned}
$$

where we have defined:

$$
\mathcal{F}(\omega, \mathbf{k})=\frac{\omega^{2}}{c^{2}}-k^{2}-\frac{4 \pi}{c^{2}} \tau \frac{e^{\star 2} \Psi_{0}^{2}}{m^{\star}}
$$

and

$$
\mathcal{G}(\omega, \mathbf{k})=\frac{\omega^{2}}{v_{s}^{2}}-\tilde{k}^{2}-4 \pi \frac{e^{\star 2} \Psi_{0}^{2}}{m^{\star} c^{2}} \frac{k_{z}^{2}}{\mathcal{F}(\omega, \mathbf{k})} \tau\left(\tau^{2}-1\right) .
$$

The remaining results are derived from the phonon equation (22)

$$
\vec{X}(\omega, \mathbf{k})=-i \frac{\delta}{\kappa} \frac{1}{\omega^{2}-\omega_{p h}^{2}}\left(\vec{q} \phi(\omega, \mathbf{k})+\frac{\omega}{c} \vec{A}(\omega, \mathbf{k})\right)
$$

and from the combination of the in-plane components of (13) and (21),

$$
\begin{aligned}
& \left(\frac{\omega^{2}}{c^{2}}-k^{2}-\frac{4 \pi}{c^{2}} \frac{e^{\star 2} \Psi_{0}^{2}}{m^{\star}}\right) \vec{A}(\omega, \mathbf{k})+\frac{\omega}{c} \vec{q} \phi(\omega, \mathbf{k}) \\
& \quad+\frac{4 \pi}{c} \frac{e^{\star} \Psi_{0}^{2}}{m^{\star}} i \vec{q} \hbar \varphi(\omega, \mathbf{k})+\frac{4 \pi}{c} i \omega \delta \vec{X}(\omega, \mathbf{k})=0 .
\end{aligned}
$$

After a straightforward calculation, we encounter that

$$
\vec{A}(\omega, \mathbf{k})=-\frac{4 \pi}{c} \omega \vec{q} \frac{\rho_{0}(\mathbf{k})}{k^{2}} \frac{\mathcal{D}(\omega, \mathbf{k})}{\mathcal{E}(\omega, \mathbf{k})} \delta\left(\omega-\omega_{p}(\mathbf{k})\right)
$$

$$
\begin{aligned}
\vec{X}(\omega, \mathbf{k})=-4 & \pi i \frac{\delta}{\kappa} \frac{\vec{q}}{k^{2}} \frac{\rho_{0}(\mathbf{k})}{\left(\omega^{2}-\omega_{p h}^{2}\right)} \delta\left(\omega-\omega_{p}(\mathbf{k})\right) \\
& \times\left[1-\frac{\omega^{2}}{c^{2}} \frac{\mathcal{D}(\omega, \mathbf{k})}{\mathcal{E}(\omega, \mathbf{k})}\right] .
\end{aligned}
$$

where we have labeled

$$
\begin{aligned}
\mathcal{D}(\omega, \mathbf{k})=1+ & 4 \pi \frac{\delta^{2}}{\kappa} \frac{1}{\omega^{2}-\omega_{p h}^{2}}-4 \pi \frac{e^{\star 2} \Psi_{0}^{2}}{m^{\star} v_{s}^{2}} \frac{1}{\mathcal{G}(\omega, \mathbf{k})} \\
& \times\left[1-\left(\tau^{2}-1\right) \frac{v_{s}^{2}}{c^{2}} \frac{k_{z}^{2}}{\mathcal{F}(\omega, \mathbf{k})}\right] \\
\mathcal{E}(\omega, \mathbf{k})=\frac{\omega^{2}}{c^{2}}- & k^{2}-\frac{4 \pi}{c^{2}} \frac{e^{\star 2} \Psi_{0}^{2}}{m^{\star}}+\frac{4 \pi}{c^{2}} \frac{(\delta \omega)^{2}}{\kappa\left(\omega^{2}-\omega_{p h}^{2}\right)} .
\end{aligned}
$$

Next, we substitute these results in the superconducting charge density definition (14)

$$
\begin{aligned}
\rho_{0}(\omega, \mathbf{k})+ & \frac{e^{\star} \Psi_{0}^{2}}{m^{\star} v_{s}^{2}}\left[\hbar i \omega \varphi(\omega, \mathbf{k})+e^{\star} \phi(\omega, \mathbf{k})\right] \\
& +i \delta \vec{q} \cdot \vec{X}(\omega, \mathbf{k})=0
\end{aligned}
$$

what yields

$$
\begin{aligned}
& \rho_{0}(\mathbf{k}) \delta\left(\omega-\omega_{p}(\mathbf{k})\right)\left\{1-\frac{4 \pi}{k^{2}} \frac{e^{\star 2} \Psi_{0}^{2}}{m^{\star} v_{s}^{2}}\left[\frac{\omega^{2}}{v_{s}^{2}} \frac{1}{\mathcal{G}(\omega, \mathbf{k})}\right.\right. \\
& \left.\quad \times\left(1-\left(\tau^{2}-1\right) \frac{v_{s}^{2}}{c^{2}} \frac{k_{z}^{2}}{\mathcal{F}(\omega, \mathbf{k})}\right)-1\right] \\
& \left.\quad+4 \pi \frac{\delta^{2}}{\kappa} \frac{1}{k^{2}} \frac{q^{2}}{\left(\omega^{2}-\omega_{p h}^{2}\right)}\left[1-\frac{\omega^{2}}{c^{2}} \frac{\mathcal{D}(\omega, \mathbf{k})}{\mathcal{E}(\omega, \mathbf{k})}\right]\right\} \\
& =0
\end{aligned}
$$

Applying the transverse gauge to A.3 and A.5,

$$
\begin{aligned}
\mathbf{k} \cdot \mathbf{A}= & -\frac{4 \pi}{c} \frac{\omega}{k^{2}} \rho_{0}(\mathbf{k}) \delta\left(\omega-\omega_{p}(\mathbf{k})\right) \\
& \times\left\{\frac { k _ { z } ^ { 2 } } { \mathcal { F } ( \omega , \mathbf { k } ) } \left[1-4 \pi \frac{e^{\star 2} \Psi^{2}}{m^{\star} v_{s}^{2}} \frac{\tau}{\mathcal{G}(\omega, \mathbf{k})}\right.\right. \\
& \left.\left.\times\left(1-\left(\tau^{2}-1\right) \frac{v_{s}^{2}}{c^{2}} \frac{k_{z}^{2}}{\mathcal{F}(\omega, \mathbf{k})}\right)\right]+q^{2} \frac{\mathcal{D}(\omega, \mathbf{k})}{\mathcal{E}(\omega, \mathbf{k})}\right\} \\
= & 0
\end{aligned}
$$

This way, we conclude that $\rho_{0}(\mathbf{k})$ is on the form

$$
\rho_{0}(\mathbf{k})=\rho(\hat{q}) \delta\left(q-q_{0}\right) \delta\left(k_{z}-k_{z 0}\right),
$$

where $q_{0}$ and $k_{z 0}$ are the zeroes of (A.8) and A.7). In the non-relativistic limit $v_{F} / c \rightarrow 0, \mathcal{F}\left(\omega_{p}, \mathbf{k}\right) \sim \mathcal{E}\left(\omega_{p}, \mathbf{k}\right) \rightarrow$ $-k^{2}$ and

$$
\mathcal{G}(\omega, \mathbf{k}) \rightarrow \frac{\omega^{2}}{v_{s}^{2}}-\tilde{k}^{2}
$$

$$
\mathcal{D}(\omega, \mathbf{k}) \rightarrow 1-4 \pi \frac{e^{\star 2} \Psi_{0}^{2}}{m^{\star}} \frac{1}{\omega^{2}-v_{s}^{2} \tilde{k}^{2}}+4 \pi \frac{\delta^{2}}{\kappa} \frac{1}{\omega^{2}-\omega_{p h}^{2}}
$$


In this limit, (A.8) and (A.7) simplify respectively to

$$
\begin{aligned}
& \left(k^{2}+4 \pi \frac{\delta^{2}}{\kappa} \frac{q^{2}}{\omega^{2}-\omega_{p h}^{2}}-4 \pi \frac{e^{\star 2} \Psi_{0}^{2}}{m^{\star}} \frac{q^{2}+\tau k_{z}^{2}}{\omega^{2}-v_{s}^{2} \tilde{k}^{2}}\right) \\
& \times \rho_{s}(\omega, \mathbf{k}) \delta\left(\omega-\omega_{p}(\mathbf{k})\right)=0 \\
& \left(k^{2}+4 \pi \frac{\delta^{2}}{\kappa} \frac{q^{2}}{\omega^{2}-\omega_{p h}^{2}}-4 \pi \frac{e^{\star 2} \Psi_{0}^{2}}{m^{\star}} \frac{\tilde{k}^{2}}{\omega^{2}-v_{s}^{2} \tilde{k}^{2}}\right) \\
& \times \rho_{s}(\omega, \mathbf{k}) \delta\left(\omega-\omega_{p}(\mathbf{k})\right)=0 .
\end{aligned}
$$

Comparing both and recalling that $\tilde{k}^{2}=q^{2}+\tau^{2} k_{z}^{2}$, then $\tau(1-\tau) k_{z}^{2}=0$. For $\tau \neq 0,1$, we immediately see that $k_{z 0}=0$. Substituting this result in one of the expressions above and integrating in $\omega$, we find that $\mathcal{D}\left(\omega_{p}, q_{0}\right)=0$.
${ }^{1}$ R. L. Withers, and J. A. Wilson, J. Phys. C: Solid State Phys. 19, 4809 (1986).

2 J. A. Wilson, and A. D. Yoffe, Adv. Phys. 18, 193 (1969); J. A. Wilson, F. J. DiSalvo and S. Mahajan, Adv. Phys. 24,117 (1975).

3 J. P. Tidman, O. Singh and A. E. Curzon, Philos. Mag. 30, 1191 (1974).

${ }^{4}$ D. A. Whitney, R. M. Fleming, and R. V. Coleman, Phys. Rev. B 15, 3405 (1977).

${ }^{5}$ R. M. Fleming, D. E. Moncton, D. B. McWhan, and F. J. DiSalvo, Phys. Rev. Lett. 45, 576 (1980).

${ }^{6}$ T. Valla, A. V. Fedorov, P. D. Johnson, J. Xue, K. E. Smith, and F. J. DiSalvo, Phys. Rev. Lett. 85, 4759 (2000).

7 T. Valla, A. V. Fedorov, P. D. Johnson, B. O. Wells, S. L. Hulbert, Q. Li, G. D. Gu and N Koshizuka, Science 285, 2110 (1999).

8 A. H. Castro Neto, Phys. Rev. Lett. 86, 4382 (2001).

${ }^{9}$ D. E. Moncton, J. D. Axe, and F. J. DiSalvo, Phys. Rev. B 16, 801 (1977).

${ }^{10}$ G. D. Mahan, in Polarons in Ionic Crystals and Polar Semiconductors, edited by J. T. Devreese (North-Holland, Amsterdam, 1971), p.553.

${ }^{11}$ C. Varma, private communication.

12 D. P. DiVincenzo, and E. J. Mele, Phys. Rev. B 29, 1685 (1984).

13 A. L. Fetter, and J. D. Walecka, Quantum Theory of ManyParticle Systems (McGraw-Hill, New York, 1971).

${ }^{14}$ K. W. -K. Shung, Phys. Rev. B 34979 (1986).

${ }^{15}$ M. Tinkham, Introduction to Superconductivity (McGrawHill, New York, 1996).

${ }^{16}$ L. Balents, M. P. A. Fisher, and C. Nayak, Int. Jou. Mod. Phys. B, 12, 1033 (1998).

17 J. Gonzalez, F. Guinea, and M. A. H. Vozmediano, Phys. Rev. Lett. 77, 3589 (1996).
18 P. Hawrylak, G. Eliasson, and J. J. Quinn, Phys. Rev. B 37, 10187 (1988).

19 J. González, F. Guinea, and M. A. H. Vozmediano, Phys. Rev. B 63, 134421 (2001).

20 N. J. Doran, B. Ricco, D. J. Titterington and G. Wexler, J. Phys. C: Solid State Phys. 11, 685 (1978).

21 J. D. Jackson, Classical Electrodynamics (John Wiley \& Sons, New York, 1975).

22 D. Pines, Elementary Excitations in Solids (AddisonWesley, Redwood City, 1963).

23 A. J. Leggett, Phys.Rev. 140 , A1869 (1965).

24 B. Uchoa, A. H. Castro Neto and G. G. Cabrera (unpublished).

25 J. Bardeen, Phys. Rev. Lett. 1, 399 (1958).

26 S. Weinberg, Nucl. Phys. B 413, 567 (1994).

27 E. Abrahams, and T. Tsuneto, Phys. Rev. 152, 416 (1966).

28 R. D. Parks, Superconductivity (Marcel Dekker Inc., New York, 1969) vol. 1, Chap. 6.

29 D. B. McWhan, J. D. Axe and R. Youngblood, Phys. Rev. B 24, 5391 (1981).

${ }^{30}$ W. L. McMillan, Phys. Rev. B 14, 1496 (1976).

31 P. Bak and D. Mukamel, Phys. Rev. B 19, 1604 (1979).

32 K. K. Fung, S. McKernan, J. W. Steeds, and J. A. Wilson, J. Phys. C:Solid State Phys. 14, 5417 (1981); C. H. Chen, J. M. Gibson and R. M. Fleming, Phys. Rev. Lett. 47, 723 (1981).

33 K. Yamaya, and T. Sambongi, J. Phys. Soc. Japan 32, 1150 (1972).

34 D. Matthey, S. Gariglio and J. -M. Triscone, Phys. Rev. Lett. 83, 3758 (2003); C. H. Ahn, S. Gariglio, P. Paruch, T. Tybell, L. Antognazza and J.-M. Triscone, Science 284, 1152 (1999). 